\title{
Subclinical Vascular Damage: Current Insights and Future Potential
}

\author{
Anna Vittoria Mattioli (iD) \\ Francesca Coppi ${ }^{2}$ \\ Antonio Manenti (iD) \\ Alberto Farinetti ${ }^{3}$ \\ 'Surgical, Medical and Dental \\ Department of Morphological Sciences \\ Related to Transplant, Oncology and \\ Regenerative Medicine, University of \\ Modena and Reggio Emilia, Modena, Italy; \\ ${ }^{2}$ Cardiology Division, Policlinico di \\ Modena, Modena, Italy; ${ }^{3}$ Department of \\ Medical and Surgical Sciences for \\ Children and Adults, University of \\ Modena and Reggio Emilia, Modena, Italy
}

Correspondence: Anna Vittoria Mattioli Surgical, Medical and Dental Department of Morphological Sciences Related to Transplant, Oncology and Regenerative Medicine University of Modena and Reggio Emilia, Via del pozzo, 7I, Modena, 4II00, Italy

Email annavittoria.mattioli@unimore.it

\begin{abstract}
The cardiovascular risk assessment must be carried out during all the different phases of life because the cardiovascular risk and the related prevention actions are dynamic and constantly evolving. As patients age, they change their exposure to various risk factors and accumulate comorbidities by changing their subjective cardiovascular risk, so it is necessary to undertake personalized early and preventive diagnostic actions. The main approach to asymptomatic vascular disease is based on primary prevention with the adoption of a healthy lifestyle. Indeed, lifestyle influences most of the traditional risk factors. In recent years, important differences between the sexes regarding cardiovascular risk factors have emerged and in particular, risk factors specific for female sex have been identified. Women are more likely to be categorized into lower risk categories for cardiovascular disease and, as a result, receive less lifestyle counseling than men, as well as less intensive prevention. This narrative review aims to analyze CVD risk prevention in asymptomatic atherosclerosis with a look at new emerging factors. In the end, we quickly analyzed the effects of the recent pandemic on lifestyle and cardiovascular risk and the potential negative effects in the long term.
\end{abstract}

Keywords: prevention, risk factors, women, lifestyle, microbiota, pandemic

\section{Introduction}

A life-long approach to cardiovascular risk (CV) is important as both prevention and risk are dynamic and continuous. Patients modify their exposure to various risk factors and accumulate comorbidities thus modifying the subjective cardiovascular risk, therefore it is necessary to undertake early and personalized diagnostic actions.

This suggests that, in addition to improving lifestyle and reducing risk factor levels in patients with established cardiovascular disease (CVD) and those at high risk of CVD, healthy people of all ages should be encouraged to adopt one healthy lifestyle since childhood.

The early diagnosis of atherosclerosis when it is not yet symptomatic is fundamental in the prevention of cardiovascular diseases. It is well known that prevention is effective: the action on lifestyle and risk factors would prevent or delay almost $80 \%$ of CVDs. ${ }^{1}$

Information on the burden of subclinical atherosclerosis provides prognostic information on the risk of atherosclerotic cardiovascular disease beyond what can be obtained from traditional risk factors alone and can improve the allocation of preventive treatment in primary prevention. Atherosclerotic cardiovascular disease takes years to clinically manifest, providing a large window for optimal management of CV prevention. ${ }^{1-3}$ Imaging technique can be used during this long symptom-free period to estimate the future risk of atherosclerotic cardiovascular disease. 
Prevention should be provided both in the general population by promoting a healthy lifestyle and, on an individual level, in individuals at moderate or high risk of CVD or in patients with established CVD, by correcting unhealthy lifestyles (eg, poor quality diet, physical inactivity, smoking) and risk factors. ${ }^{3,4}$

In clinical practice, the preventive intervention during the life of a patient is divided into two phases: before and after the first symptomatic events, in which primary and secondary prevention strategies are applicable. ${ }^{5}$

The objective of this review is to analyze the established and the new strategies for the prevention of cardiovascular diseases. These strategies should be introduced from the asymptomatic phase of atherosclerotic disease.

\section{Primary Prevention of Cardiovascular Disease}

The prevention of cardiovascular diseases is defined as a coordinated action aimed at eliminating or minimizing the impact of cardiovascular diseases and related disabilities. It can be done either at the population level or customized on the individual. ${ }^{1}$

Traditional risk factors are well known and can be prevented through a healthy lifestyle. ${ }^{6}$ They include dyslipidemia, high LDL cholesterol, low HDL cholesterol, hypertriglyceridemia, sedentary lifestyle, obesity, hypertension, smoking, diabetes.

In addition, stress, depression, and low socio-economic level act by promoting unhealthy diet and low physical activity (Table 1$)^{6}$

A common mechanism of several risk factors is inflammation (Figure 1).

The histological study shows a high density of bloodborne immune and inflammatory cell infiltrates in atheroma.

Several immune cells have been identified within the atheroma including T cells, neutrophils, macrophages and mast cells, which show signs of activation and produce pro-inflammatory interleukins and cytokines. ${ }^{7}$ Despite the clear link between inflammation and atherogenesis, the causal relationship remains uncertain. ${ }^{8}$

The most common biomarker of inflammation is highsensitivity C-reactive protein (hs-CRP). To date, due to the relationship between endothelial damage, inflammation and atherosclerosis, the measurement of hs-CRP is essential in evaluating cardiovascular risk and is an important
Table I Traditional Risk Factors Affecting Cardiovascular Risk and Women-Specific Risk Factors

\begin{tabular}{|l|}
\hline (A) Traditional risk factors affecting both men and women \\
\hline Traditional risk factors \\
Dyslipidemia (including cholesterol and triglycerides) \\
Diabetes \\
Hypertension \\
Obesity and overweight \\
Physical inactivity and sedentary lifestyle \\
Stress \\
Smoking \\
Unhealthy diet \\
Homocysteine \\
Socio-economic status \\
\hline (B) Specific risk factors for women at different life stages \\
\hline Women-specific risk factors during lifetime \\
Young women \\
- PCOS \\
- Menarche \\
- Pre-term menopause \\
\hline Pregnancy \\
- Hypertensive disorders of pregnancy \\
- Gestational diabetes \\
- Preterm delivery \\
- Persisting overweight after delivery \\
\hline Adult and old women \\
- Menopause \\
- Depression \\
- Autoimmune disease \\
\hline
\end{tabular}

prognostic factor for acute myocardial infarction, stroke, peripheral arterial disease, atrial fibrillation and cardiac insufficiency. Additionally, hs-CRP can be used to assess the risk of recurrence of cardiac events in patients with stable coronary artery disease and myocardial infarction. However, the evaluation of hs-CRP has some limitations due to the high variability between subjects and to the influence of various other factors. ${ }^{9-12}$

If the usefulness of hs-CRP is well defined in the highrisk patient, much more controversial is its use in primary prevention. ${ }^{9-12}$ It is recommended in patients at moderate risk of coronary artery disease to determine the need for further evaluation, the timing of follow-up visits and drug treatment. Moreover, some evidence suggests that it may be a useful biomarker in risk assessment in overweight, prediabetic and diabetic patients. 

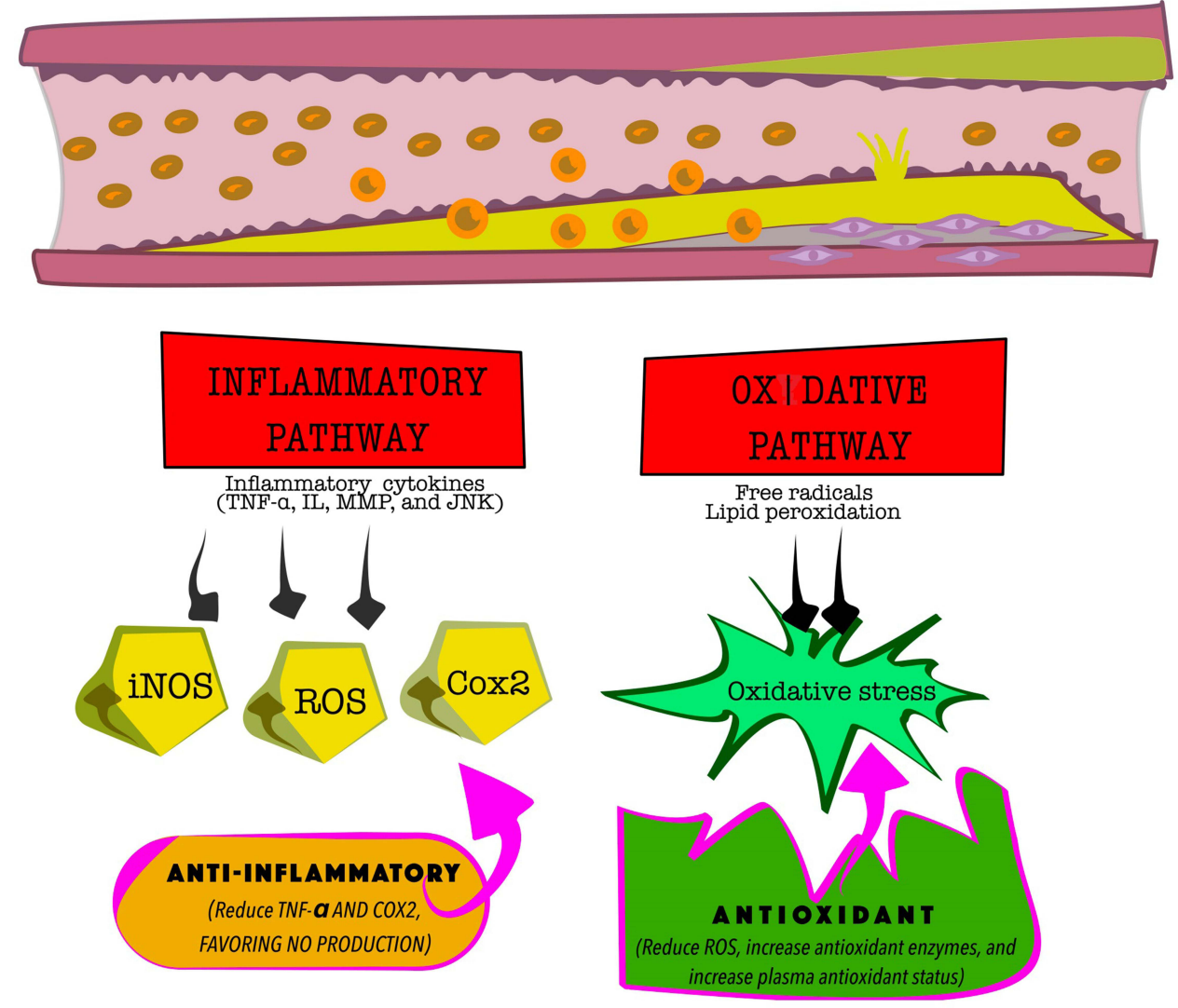

Figure I The main mechanisms involved in the development of atherosclerosis.

Abbreviations: iNOS, nitric oxide synthase; ROS, reactive oxygen species; COX-2, cyclooxygenase 2.

\section{Lifestyle and Risk Factors}

Primary prevention focuses on the non-pharmacological treatment of risk factors that is achieved through the adoption of a healthy lifestyle ${ }^{1,2,13}$ (Box 1).

Lifestyle is the sum of actions driven by several interconnected environmental factors and their consequences on health. Unhealthy lifestyle includes the interplay between an unhealthy diet containing food rich in sugar and fat, sedentary habits and reduced physical activity, increasing consumption of cigarettes and alcohol, high levels of stress, psychological disturbances and poor sleep. ${ }^{14}$

The combination of these behaviors leads to obesity, which is a well-known and studied cardiovascular risk factor. Obesity, especially visceral obesity, is associated with high levels of inflammation as are dyslipidemia, hypertension and diabetes. ${ }^{15,16}$

Obesity is characterized by dysfunctional adipose tissue; during periods of caloric excess the adipocytes initially become hypertrophic and secrete adipokines, which determine the recruitment of further pre-adipocytes, which evolve into mature adipocytes as compensatory protection mechanism. Fat distribution also influenced atherosclerosis. The visceral fat is associated with the metabolic syndrome, its associated CVD risk factors, and an increased risk of clinical CVD. In addition, visceral adiposity plays a central role by affecting insulin resistance. ${ }^{18}$

The effects of a healthy diet on various risk factors are known and well established. Diets rich in antioxidant foods (eg, Mediterranean and DASH diets) have a protective action on the cardiovascular system. Among the different diets the most studied is the Mediterranean diet. ${ }^{19,20}$

Recently, Dinu and collaborators analyzed a large number of meta-analyses that evaluated the effects of several popular diets on weight and cardiometabolic risk factors and concluded that the Mediterranean diet had the strongest and most consistent evidence of efficacy, with no harmful effects. Also for the DASH diet, it was found to be effective in reducing body weight and blood pressure.

On the contrary, diets with low carbohydrate content, high protein content, low fat content and low glycemic index/load, having beneficial effects on weight loss, have 
Box I Practical Suggestions for Primary Prevention of Preclinical Atherosclerosis

Evaluation of 10 -year atherosclerotic CVD risk estimation

Discuss with clinician before starting on pharmacologic therapy

Consume a healthy diet rich of vegetables, fruits, whole grains, lean vegetable or animal protein, and fish. Minimizes the intake of red meat and processed red meats, refined carbohydrates, and sweetened beverages.

Engage in at least 150 minutes per week of accumulated moderate-intensity physical activity or 75 minutes per week of vigorous-intensity physical activity

Reduce sitting time and screen time (ie, watching TV)

Avoid tobacco use

highlighted potential risks of unfavorable changes in lipid, glycemic or blood pressure parameters. ${ }^{21}$

MedD has been shown to be effective in reducing cardiovascular mortality, in controlling arterial hypertension, in preventing obesity and metabolic syndrome. ${ }^{22,23}$

A systematic review that analyzed data from 7186 subjects reported a significant reduction in visceral obesity on a Mediterranean or similar diet. ${ }^{24}$

The Mediterranean diet is rich in fruit and vegetables and is naturally low in sodium due to its low degree of processing foods. ${ }^{19,21,22}$ Diets rich in fruit and vegetables, such as the Mediterranean diet, have several positive effects on the vascular system, especially for the antioxidant action, the increase in nitric oxide species and the reduction of inflammation. ${ }^{25,26}$

These beneficial effects are quite similar in men and women although adherence to diet and healthy lifestyle are different in the sexes. ${ }^{14,27}$

Regular physical activity is also a key part of a healthy lifestyle. The fight against a sedentary lifestyle and the promotion of regular physical activity are important in correcting almost all cardiovascular risk factors. ${ }^{28}$ Regular physical activity (PA) reduces inflammation and endothelial dysfunction. ${ }^{28}$

Fat loss mediated by changes in diet, exercise, and/or surgery is associated with decreased markers of adipose tissue and systemic inflammation. ${ }^{29-31}$

Weight loss achieved through low-calorie diet and/or exercise resulted in a decrease in IL-6, CRP, TNF $\alpha$, MCP1, soluble intercellular adhesion molecule-1 (ICAM-1) and cell adhesion molecule vascular-1 (VCAM-1) ${ }^{32}$

In most countries, most adults and children do not reach the minimum levels of activity recommended by health organizations: each person should engage in moderate exercise for at least 150 minutes per week and/or vigorous activity for at least 75 minutes per week or an equivalent thereof. ${ }^{1,33}$

For population-based prevention, the "seven best investments" declaration offers universal and comprehensive advice to promote PA. ${ }^{34}$

Recent campaigns by sports medicine societies have approved prescriptions for the PA (http://www.efsma.net) and have suggested that PA levels be assessed at each medical visit. Additionally, the amount of sedentary time should be minimized by actively traveling (cycling or walking), taking breaks from long sitting periods, and reducing screen time. Short, frequent exercises are more beneficial and affordable than occasional supervised exercise classes at the gym. ${ }^{35}$

This aspect is particularly relevant for women. Women are less likely to engage in regular physical activity due to different social roles: at work and in the family as a result of traditional social roles and stereotypes. ${ }^{36}$ Achieving healthy eating and constant physical activity adds to work and family activities and is time consuming. All this implies a big commitment in terms of time and costs. $^{37}$

\section{Lifestyle and Risk Factors in Women}

Although cardiovascular disease (CVD) remains the leading cause of death among men and women, women are still underdiagnosed and may experience a delay in diagnosis. ${ }^{38,39}$ Furthermore, in addition to traditional $\mathrm{CV}$ risk factors, there are sex-specific risk factors, which contribute to the risk profile in women ${ }^{38,39}$ (Table 1). The main risk factor in a woman's lifespan is menopause. $^{40}$

Indeed, several scientific evidence supports the idea that the different impacts of cardiovascular disease and differences in vascular biology in men and women may 
be related to the effects of sex steroid hormones on the cardiovascular and metabolic systems. ${ }^{40}$

The gap in the diagnosis and treatment of cardiovascular disease in women depends on several factors: the lack of self-perception of cardiovascular risk, the exposure to risk factors that act with a difference in women and men, and specific sex-related risk factors, which are still little known. ${ }^{41}$

Sexual dimorphism in fat distribution and metabolism of adipose tissue and skeletal muscle affects sex differences in tissue-specific insulin sensitivity and cardiometabolic health. Additionally, sex hormones, such as estrogen and testosterone, as well as the complement of sex chromosomes, are likely to play a role in sex differences in cardiovascular disease. $^{27,42}$

Women have less obstructive coronary artery disease than men across the entire spectrum of acute coronary syndromes and across all age groups. ${ }^{43-45}$

Furthermore, women have better peripheral endothelial function than men at least up to the age of 70 and regardless of exposure to various risk factors. ${ }^{46-48}$

Improved vascular function has also been reported in women undergoing coronary angiography but without obstructive coronary artery disease. Han et al found that women had less diffuse epicardial endothelial dysfunction than men. ${ }^{49}$ Sex hormones affect endothelial function through their effects on agonists and contribute to functional differences between women and men in relation to endothelial function. In women, estrogen promotes NO release via endothelial NO synthase (eNOS) activation, while androgen receptor engagement may result in altered endothelial release of $\mathrm{NO} .^{50}$

These differences between men and women pose the problem of thinking about different strategies for vascular prevention. Personalized prevention not only based on age and traditional risk factors but also on the basis of a woman's gender and specific risk factors.

This thought has led to a different approach as is evident in the most recent literature. ${ }^{51}$

It has been suggested that some specific risk factors could be used as indicators of increased CV risk and the likelihood of developing $\mathrm{CV}$ events in the future life. Gestational diabetes, hypertension disorders during gestation, eclampsia could be used as indicators of an increased $\mathrm{CV}$ risk being linked to endothelial dysfunction and altered vascular response. These individuals should undergo periodic cardiovascular risk assessment even before menopause. $^{36,39}$
Stress and depression are major risk factors for women. These conditions promote changes in lifestyle, switching to unhealthy diet, eat-drink for cope with stress, and sedentary behavior. Women were more likely to develop eating for coping and food craving to cope with stress. ${ }^{14}$ Food craving is characterized by a high intake of fat- and sugar-rich foods. ${ }^{14}$ During the recent pandemic, the economic crisis mainly affected women who were the most exposed, aggravating situations of stress and depression. This is likely to lead to an increase in $\mathrm{CV}$ disease in women over the next few years.

\section{Future Developments Role of Microbiota}

An unusual point of view in cholesterol control considers the role of the microbiota.

The gut microbiota is an important environmental factor and there is increasingly concrete evidence that the activity and composition of the gut microbiota are closely related to health. ${ }^{52,53}$

The gut microbiota is hypothesized to participate in nutrient and energy uptake, modulate the innate immune system, and produce a wide variety of small-molecule metabolites that are detected by host receptor systems to regulate host metabolism and inflammatory pathways linked to CVD. ${ }^{52,53}$

In addition to direct interactions, the gut microbiota can indirectly interact with the host via metabolites, which can appear in the circulation and in the urine.

There is a strong relationship between microbiota and CV risk factors such as obesity: it has been seen that the diversity of the intestinal microbiota in obese patients is lower than that of the normal weight population and this decrease can lead to an increase in insulin resistance. ${ }^{54-56}$

A further link is that the microbiota is strongly influenced by the diet and the diet plays a central role in the development of many $\mathrm{CV}$ risk factors. ${ }^{57} \mathrm{~A}$ biologically active metabolite of the gut microbiota, which appears to be involved in the development of atherosclerosis, is trimethylamine N-oxide (TMAO).

The increase in plasma TMAO level is correlated with lipid deposition in blood vessels and endothelial dysfunction. ${ }^{58}$ TMAO can also impair reverse cholesterol transport. $^{59}$

Studies have revealed the association of altered microbiota with increased triglyceride levels, decreased highdensity lipoprotein cholesterol (HDL-c) and slightly 
modified LDL-c level. ${ }^{60}$ Two studies of obese and healthy European individuals found that elevated fasting triglycerides and diminished HDL-c are associated with the low abundance of the gut microbiota in fasting triglycerides and a decrease in HDL-c is associated with the low abundance of the intestinal microbiota. ${ }^{61,62}$

Furthermore, following the results observed in patients in the LifeLines-DEEP study regarding blood lipid levels, body mass index and gut microbiota, it has been suggested that the gut microbiota may affect only certain aspects of lipid metabolism and distinct classes of lipoproteins, such as HDL and very low-density lipoprotein (VLDL). ${ }^{63}$ Since the microbiota is heavily influenced by diet, a healthy lifestyle could also act through it. Several evidences underline that the drugs normally used in secondary prevention such as statins and new-generation oral hypoglycemic agents also act by modifying the intestinal microbiota. ${ }^{57,61,63}$

\section{Role of Mitochondrial Dysfunction}

In recent years, a growing number of studies have shown that atherosclerosis may be related to mitochondrial fusion and fission. ${ }^{64}$

In recent years, a growing number of studies have shown that atherosclerosis may be related to mitochondrial fusion and fission. ${ }^{64}$

During the early stages of atherosclerosis, increased ROS levels induce endothelial dysfunction, vascular inflammation, and accelerated accumulation of oxidized low-density lipoprotein (ox-LDL) in the arterial wall. Excessive LDL deposition can induce apoptosis of vascular cells through a dependent mitochondrial pathway after oxidative modification. Furthermore, increased ROS levels cause mitochondrial dysfunction in vascular cells and proliferation of smooth muscle cells, and are responsible for inducing the development of vascular atherosclerosis. ${ }^{65} \mathrm{In}$ addition to the control of lifestyle and risk factors and drugs such as statins, new types of antioxidants and mitochondrial regulators such as mdivi-1 have become research hot spots for the treatment of atherosclerosis.

Furthermore, angiogenesis has recently played a role in the therapy of cardiovascular disease. Restoration of intravascular perfusion by improving or inhibiting angiogenesis is an innovative approach in the treatment of peripheral arterial disease (PAD) caused by atherosclerosis. ${ }^{66}$

Mitochondria seems to play a key role in angiogenic responses induced by growth factors such as VEGF by regulating mitoROS-related activities. ${ }^{67}$
Drugs targeting selective mitochondria such as mitochondrial antioxidants are being evaluated in preclinical and clinical studies. Resveratrol has been shown to promote mitochondrial fusion and can improve endothelial cells by reducing ROS and maintaining mitochondrial membrane proteins and could be used in the prevention of atherosclerosis. ${ }^{68,69}$

\section{Role of Epigenetic-Sensitive Mechanisms}

Epigenetic-sensitive mechanisms are involved in the onset and development of atherosclerosis and could play a potential role as non-invasive biomarkers. The epigenome includes a large spectrum of molecular changes leading to a functional reinterpretation of DNA sequence by dynamic activation/repression of specific genes, without changing the nucleotide sequence. ${ }^{70-72}$ The main epigenetic determinants are DNA and mRNA methylation, post-translational histone/non-histone modifications, and non-coding RNA. ${ }^{70-72}$ DNA methylation is the most common form of epigenetic modification and involves the addition of methyl (-CH3) groups to cytosine-phosphate-guanine $(\mathrm{CpG})$ sites of genes. Generally, increased methylation in gene promoters regions of DNA that regulate gene expression via activation of transcription is associated with decreased gene expression, and decreased methylation is associated with increased gene expression. ${ }^{73}$ The cell-specific patterns of epigenetic modifications, mainly DNA and RNA methylation are affected by environmental exposure. One hypothesis is that the onset of atherosclerosis may be explained by the mechanism of "persistent memory", for which early environmental injuries experienced during prenatal or perinatal life are translated in epigenetic changes inherited across several generations. ${ }^{74,75}$ This mechanism is defined as "transgenerational epigenetic inheritance", and several preclinical studies support it. ${ }^{74,75}$ Clinical epigenetics (mainly DNA methylation) would be able to unveil new pathogenetic mechanisms and validate new artificial intelligence algorithms capable of predicting high-risk patients for subclinical atherosclerosis.

\section{Impact of COVID-19 Pandemic}

After the Spagnola pandemic throughout 1918, a spike in cardiovascular events was reported, with deaths caused by cardiovascular events in excess of deaths from other causes, including pneumonia. ${ }^{76} \mathrm{We}$ must be prepared to face the likely increase in cardiovascular events soon after the end of the Covid-19 pandemic. 
The recent COVID-19 pandemic has greatly affected not only mental health but also the physical health of patients. ${ }^{77-79}$ Most of the subjects in primary prevention have reduced and/or eliminated visits to clinic, have adopted unhealthy lifestyles by reducing outdoors physical activity and have changed eating habits, in some cases as a way to cope with stress. ${ }^{80,81}$ Changes towards unhealthy lifestyles were related to quarantine and restrictions induced by Governments and to pandemic-related stress. $^{77,81}$

Moreover, a clinical condition called "long COVID" is currently emerging. Long COVID identified the individuals that do not recover for several weeks or months following the onset of symptoms suggestive of COVID19. Women seem to be twice as likely to develop long COVID as men, but only until around age 60 , when the risk level becomes similar.

The cause for the symptoms of long COVID may be an extreme inflammatory response triggered by the virus, but also an autoimmune reaction - unveiled\| by the virus itself. ${ }^{77,82}$ The adverse pathophysiological responses induced by the virus SARS-CoV-2 on the CV system, in combination with other factors, such as age, sex, cardiovascular and metabolic comorbidities: increase the risk of cardiac and vascular injury. Furthermore, some therapeutic protocols adopted to fight against the virus can cause cardiotoxicity. Different classes of drugs present in the treatment COVID-19 may increase the risk of heart and vascular injury leading to long COVID. ${ }^{82}$ The prospects for the evolution of COVID-19 systemic pathology are aimed at evaluating long-term degenerative effects and sequelae.

After pandemic, it will be necessary to re-evaluate patients according to their individual $\mathrm{CV}$ risk with particular attention to those affected by long COVID. Support can come from imaging methods. In addition to traditional risk assessment models, subclinical atherosclerosis imaging modalities personalize future ASCVD risk stratification and guide the initiation and intensification of preventive therapies. There are several emerging imaging techniques, but more research is needed for clinical use in the primary prevention.

\section{Abbreviations}

$\mathrm{CV}$, cardiovascular; CVD, cardiovascular disease; LDL cholesterol, low-density lipoprotein cholesterol; HDL cholesterol, high-density lipoprotein cholesterol; hs-CRP, high-sensitivity C-reactive protein; PA, physical activity; PCOS, polycystic ovary syndrome.

\section{Acknowledgments}

We thank Dr Melania Malpezzi for the production of Figure 1.

\section{Author Contributions}

All authors made a significant contribution to the work reported, whether that is in the conception, study design, execution, acquisition of data, analysis and interpretation, or in all these areas; took part in drafting, revising or critically reviewing the article; gave final approval of the version to be published; have agreed on the journal to which the article has been submitted; and agree to be accountable for all aspects of the work.

\section{Funding}

This research received no specific grant from any funding agency in the public, commercial, or not-for-profit sectors.

\section{Disclosure}

The authors report no conflicts of interest in this work.

\section{References}

1. Piepoli MF, Frank L, Visseren J, et al. ESC Scientific Document Group, 2021 ESC Guidelines on cardiovascular disease prevention in clinical practice. Eur Heart J. 2021;42(34):3227-3337. doi:10.1093/ eurheartj/ehab484

2. Kotseva K, Wood D, De Bacquer D, et al. EUROASPIRE IV: a European Society of Cardiology survey on the lifestyle, risk factor and therapeutic management of coronary patients from 24 European countries. Eur J Prev Cardiol. 2016;23:636-648.

3. Aboyans V, Ricco JB, Bartelink MEL; ESC Scientific Document Group. 2017 ESC Guidelines on the Diagnosis and Treatment of Peripheral Arterial Diseases, in collaboration with the European Society for Vascular Surgery (ESVS): document covering atherosclerotic disease of extracranial carotid and vertebral, mesenteric, renal, upper and lower extremity arteriesEndorsed by: the European Stroke Organization (ESO)The Task Force for the Diagnosis and Treatment of Peripheral Arterial Diseases of the European Society of Cardiology (ESC) and of the European Society for Vascular Surgery (ESVS). Eur Heart J. 2018;39(9):763-816. doi:10.1093/eurheartj/ ehx095

4. D'Ascenzi F, Sciaccaluga C, Cameli M, et al. When should cardiovascular prevention begin? The importance of antenatal, perinatal and primordial prevention. Eur J Prev Cardiol. 2019;16:2047487319893832. doi:10.1177/2047487319893832

5. Ahmadi A, Argulian E, Leipsic J, Newby DE, Narula J. From subclinical atherosclerosis to plaque progression and acute coronary events: JACC state-of-the-art review. J Am Coll Cardiol. 2019;74 (12):1608-1617. doi:10.1016/j.jacc.2019.08.012

6. Kotseva K, Wood D, De Backer G, et al. EUROASPIRE III. Management of cardiovascular risk factors in asymptomatic high-risk patients in general practice: cross-sectional survey in 12 European countries. Eur J Cardiovasc Prev Rehabil. 2010;17:530-540. 
7. Skålén K, Gustafsson M, Rydberg EK, et al. Subendothelial retention of atherogenic lipoproteins in early atherosclerosis. Nature. 2002;417 (6890):750-754. doi:10.1038/nature00804

8. Shaya GE, Leucker TM, Jones SR, Martin SS, Toth PP. Coronary heart disease risk: low-density lipoprotein and beyond. Trends Cardiovasc Med. 2021. doi:10.1016/j.tcm.2021.04.002

9. Carrero JJ, Franko MA, Obergfell A, Gabrielsen A, Jernberg T. hsCRP level and the risk of death or recurrent cardiovascular events in patients with myocardial infarction: a healthcare-based study. $J \mathrm{Am}$ Heart Assoc. 2019;8:e12638.

10. Yoshikawa T, Hata J, Sakata S, et al. Serum high-sensitivity C-reactive protein levels and the development of atrial fibrillation in a general Japanese Population - the Hisayama study. Circ J. 2021;85(8):1365-1372. doi:10.1253/circj.CJ-20-0751

11. Ziv-Baran T, Shenhar-Tsarfaty S, Etz-Hadar I, et al. The ability of the wide range CRP assay to classify individuals with low grade inflammation into cardiovascular risk groups. Clinica Chimica Acta. 2017;471:185-190. doi:10.1016/j.cca.2017.06.008

12. Su H, Pei Y, Tian C, et al. Relationship between high-sensitivity C-reactive protein and subclinical carotid atherosclerosis stratified by glucose metabolic status in Chinese adults. Clin Cardiol. 2019;42(1):39-46. doi:10.1002/clc.23095

13. Betageri O, Al-Turk B, Freeman AM, Aggarwal M. Prevention and reversal of morbidity in today's cardiovascular patient: role of lifestyle modification and nutrition in the current era. Curr Cardiol Rep. 2021;23(10):143. doi:10.1007/s11886-021-01577-1

14. Mattioli AV, Sciomer S, Maffei S, Gallina S. Lifestyle and stress management in women during COVID-19 pandemic: impact on cardiovascular risk burden. Am J Lifestyle Med. 2020;15(3):356359. PMID: 34025328. doi:10.1177/1559827620981014

15. Goossens GH, Blaak EE. Adipose tissue dysfunction and impaired metabolic health in human obesity: a matter of oxygen? Front Endocrinol. 2015;6:55. doi:10.3389/fendo.2015.00055

16. Lavie CJ, Milani RV, Ventura HO. Obesity and cardiovascular disease: risk factor, paradox, and impact of weight loss. $J$ Am Coll Cardiol. 2009;53:1925-1932. doi:10.1016/j.jacc.2008.12.068

17. Mattioli AV, Pinti M, Farinetti A, Nasi M. Obesity risk during collective quarantine for the COVID-19 epidemic. Obes Med. 2020;20:100263. doi:10.1016/j.obmed.2020.100263

18. Després JP, Carpentier AC, Tchernof A, Neeland IJ, Poirier P. Management of obesity in cardiovascular practice: JACC focus seminar. J Am Coll Cardiol. 2021;78(5):513-531. doi:10.1016/j.jacc.2021.05.035

19. Mattioli AV, Migaldi M, Farinetti A. Coffee in hypertensive women with asymptomatic peripheral arterial disease: a potential nutraceutical effect. J Cardiovasc Med. 2018;19:183-185.

20. Dinu M, Pagliai G, Lotti S, Giangrandi I, Colombini B, Sofi F. Adherence to Mediterranean diet measured through medi-lite score and obesity: a retrospective study. Nutrients. 2021;13(6):2007. PMID: 34200879; PMCID: PMC8230530. doi:10.3390/nu13062007

21. Dinu M, Pagliai G, Angelino D, et al. Effects of popular diets on anthropometric and cardiometabolic parameters: an umbrella review of meta-analyses of randomized controlled trials. Adv Nutr. 2020;11 (4):815-833. doi:10.1093/advances/nmaa006

22. Mattioli AV, Coppi F, Migaldi M, Scicchitano P, Ciccone MM, Farinetti A. Relationship between Mediterranean diet and asymptomatic peripheral arterial disease in a population of pre-menopausal women. Nutr Metab Cardiovasc Dis. 2017;27(11):985-990.

23. Bakaloudi DR, Chrysoula L, Kotzakioulafi E, Theodoridis X, Chourdakis M. Impact of the level of adherence to Mediterranean diet on the parameters of metabolic syndrome: a systematic review and meta-analysis of observational studies. Nutrients. 2021;13 (5):1514. doi:10.3390/nu13051514

24. Magriplis E, Panagiotakos D, Kyrou I, et al. Presence of hypertension is reduced by Mediterranean diet adherence in all individuals with a more pronounced effect in the obese: the Hellenic National Nutrition and Health Survey (HNNHS). Nutrients. 2020;12:853.
25. Bendall CL, Mayr HL, Opie RS, Bes-Rastrollo M, Itsiopoulos C, Thomas CJ. Central obesity and the Mediterranean diet: a systematic review of intervention trials. Crit Rev Food Sci Nutr. 2018;58 (18):3070-3084. doi:10.1080/10408398.2017.1351917

26. Siti HN, Kamisaha Y, Kamsiaha J. The role of oxidative stress, antioxidants and vascular inflammation in cardiovascular disease (a review). Vascul Pharmacol. 2015;71:40-56.

27. Goossens GH, Jocken JWE, Blaak EE. Sexual dimorphism in cardiometabolic health: the role of adipose tissue, muscle and liver. Nat Rev Endocrinol. 2021;17(1):47-66. doi:10.1038/ s41574-020-00431-8

28. Nasi M, Patrizi G, Pizzi C, et al. The role of physical activity in individuals with cardiovascular risk factors: an opinion paper from Italian Society of Cardiology-Emilia Romagna-Marche and SICSport. J Cardiovasc Med. 2019;20(10):631-639. doi:10.2459/ JCM.0000000000000855

29. Chait A, den Hartigh LJ. Adipose tissue distribution, inflammation and its metabolic consequences, including diabetes and cardiovascular disease. Front Cardiovasc Med. 2020;7:22. doi:10.3389/ fcvm.2020.00022

30. Forsythe LK, Wallace JM, Livingstone MB. Obesity and inflammation: the effects of weight loss. Nutr Res Rev. 2008;21:117-133. doi: $10.1017 / \mathrm{S} 0954422408138732$

31. Selvin E, Paynter NP, Erlinger TP. The effect of weight loss on Creactive protein: a systematic review. Arch Intern Med. 2007;167:3139. doi:10.1001/archinte.167.1.31

32. Madsen EL, Rissanen A, Bruun JM, et al. Weight loss larger than $10 \%$ is needed for general improvement of levels of circulating adiponectin and markers of inflammation in obese subjects: a 3year weight loss study. Eur J Endocrinol. 2008;158:179-187. doi:10.1530/EJE-07-0721

33. Pelliccia A, Sharma S, Gati S; ESC Scientific Document Group, et al. 2020 ESC Guidelines on sports cardiology and exercise in patients with cardiovascular disease: the Task Force on sports cardiology and exercise in patients with cardiovascular disease of the European Society of Cardiology (ESC). Eur Heart J. 2021;42(1):17-96. doi:10.1093/eurheartj/ehaa605.

34. Global Advocacy Council for Physical Activity, International Society for Physical Activity and Health. The Toronto Charter for Physical Activity: a global call for action. J Phys Activity Health. 2010;7 (Suppl3):S370-S385.

35. Garrett S, Elley CR, Rose SB, O'Dea D, Lawton BA, Dowell AC. Are physical activity interventions in primary care and the community cost-effective? A systematic review of the evidence. $\mathrm{Br} J \mathrm{Gen}$ Pract. 2011;61:e125-e133.

36. Sciomer S, Moscucci F, Maffei S, Gallina S, Mattioli AV. Prevention of cardiovascular risk factors in women: the lifestyle paradox and stereotypes we need to defeat. Eur J Prev Cardiol. 2019;26(6):609610. doi: $10.1177 / 2047487318810560$

37. Coppi F, Nasi M, Farinetti A, et al. Physical activity, sedentary behaviour, and diet in menopausal women: comparison between COVID19 "first wave" and "second wave" of pandemic in Italy. Prog Nutri. 2021;23(2):11755.

38. Garcia M, Mulvagh SL, Merz CN, Buring JE, Manson JE. Cardiovascular disease in women: clinical perspectives. Circ Res. 2016;118(8):1273-1293.

39. Mattioli AV, Sciomer S, Moscucci F, et al. Cardiovascular prevention in women: a narrative review from the Italian Society of Cardiology working groups on 'Cardiovascular Prevention, Hypertension and peripheral circulation' and on 'Women Disease'. J Cardiovasc Med. 2019;20(9):575-583.

40. Matthews KA, Crawford SL, Chae CU, et al. Are changes in cardiovascular disease risk factors in midlife women due to chronological aging or to the menopausal transition? $J$ Am Coll Cardiol. 2009;54:2366-2373. 
41. Cocchi C, Coppi F, Farinetti A, Mattioli AV. Cardiovascular disease prevention and therapy in women with Type 2 diabetes. Future Cardiol. 2021. doi:10.2217/fca-2021-0011

42. Woodward M. Cardiovascular disease and the female disadvantage. Int J Environ Res Public Health. 2019;16:1165.

43. Paolisso P, Bergamaschi L, Saturi G, et al. Prevention medical therapy and outcomes in patients with myocardial infarction with nonobstructive coronary artery disease. Front Pharmacol. 2020;10:1606. doi:10.3389/fphar.2019.01606

44. Berger JS, Elliott L, Gallup D, et al. Sex differences in mortality following acute coronary syndromes. JAMA. 2009;302:874-882.

45. Rosengren A, Wallentin L, Gitt AK, Behar S, Battler A, Hasdai D. Sex, age, and clinical presentation of acute coronary syndromes. Eur Heart J. 2004;25:663-670.

46. Benjamin EJ, Larson MG, Keyes MJ, et al. Clinical correlates and heritability of flow-mediated dilation in the community: the Framingham Heart Study. Circulation. 2004;109:613-619.

47. Celermajer D, Sorensen K, Bull C, Robinson J, Deanfield J. Endothelium-dependent dilation in the systemic arteries of asymptomatic subjects relates to coronary risk factors and their interaction. $J$ Am Coll Cardiol. 1994;24:1468-1474.

48. Juonala M, Kahonen M, Laitinen T, et al. Effect of age and sex on carotid intima-media thickness, elasticity and brachial endothelial function in healthy adults: the Cardiovascular Risk in Young Finns Study. Eur Heart J. 2008;29:1198-1206.

49. Han SH, Bae JH, Holmes DR Jr, et al. Sex differences in atheroma burden and endothelial function in patients with early coronary atherosclerosis. Eur Heart J. 2008;29:1359-1369.

50. Stanhewicz AE, Wenner MM, Stachenfeld NS. Sex differences in endothelial function important to vascular health and overall cardiovascular disease risk across the lifespan. Am J Physiol Heart Circ Physiol. 2018;315(6):H1569-H1588. doi:10.1152/ajpheart.00396.2018

51. Meadows JL, Shah S, Burg MM, Pfau S, Soufer R. Cardiovascular imaging of biology and emotion: considerations toward a new paradigm. Circ Cardiovasc Imaging. 2020;13(8):e011054. PMID:32762255. doi:10.1161/CIRCIMAGING.120.011054

52. Clemente JC, Ursell LK, Parfrey LW, Knight R. The impact of the gut microbiota on human health: an integrative view. Cell. 2012;148:1258-1270. doi:10.1016/j.cell.2012.01.035

53. Ding Q-Y, Tian J-X, Li M, et al. Interactions between therapeutics for metabolic disease, cardiovascular risk factors, and gut microbiota. Front Cell Infect Microbiol. 2020;10:530160. doi:10.3389/fcimb.2020.530160

54. Wolf G. Gut microbiota: a factor in energy regulation. Nutr Rev. 2006;64:47-50. doi:10.1111/j.1753-4887.2006.tb00173.x

55. Jiao N, Baker SS, Nugent CA, et al. Gut microbiome may contribute to insulin resistance and systemic inflammation in obese rodents: a meta-analysis. Physiol Genomics. 2018;50:244-254. doi:10.1152/ physiolgenomics.00114.2017

56. Jie Z, Xia H, Zhong SL, et al. The gut microbiome in atherosclerotic cardiovascular disease. Nat Commun. 2017;8:845. doi:10.1038/ s41467-017-00900-1

57. Kirichenko TV, Markina YV, Sukhorukov VN, et al. Insight at atherogenesis: the role of microbiome. Front Cell Dev Biol. 2020;8:586189. doi:10.3389/fcell.2020.586189

58. Hardin SJ, Singh M, Eyob W, et al. Diet-induced chronic syndrome, metabolically transformed trimethylamine-N-oxide, and the cardiovascular functions. Rev Cardiovasc Med. 2019;20:121-128. doi:10.31083/j.rcm.2019.03.518

59. Sun X, Jiao X, Ma Y, et al. Trimethylamine N-oxide induces inflammation and endothelial dysfunction in human umbilical vein endothelial cells via activating ROS-TXNIP-NLRP3 inflammasome. Biochem Biophys Res Commun. 2016;481:63-70.

60. Fisher EA, Feig JE, Hewing B, Hazen SL, Smith JD. High density lipoprotein function, dysfunction, and reverse cholesterol transport. Arterioscler Thromb Vasc Biol. 2012;32:2813-2820. doi:10.1161/ ATVBAHA.112.300133
61. Cotillard A, Kennedy SP, Kong LC, et al. Dietary intervention impact on gut microbial gene richness. Nature. 2013;500:585-588. doi:10.1038/nature12480

62. Nielsen T, Qin J, Prifti E, et al. Richness of human gut microbiome correlates with metabolic markers. Nature. 2013;500:541-546. doi:10.1038/nature12506

63. Kurilshikov A, van den Munckhof ICL, Chen L, et al. Gut microbial associations to plasma metabolites linked to cardiovascular phenotypes and risk. Circ Res. 2019;124(12):1808-1820. doi:10.1161/ CIRCRESAHA.118.314642

64. Li D, Yang S, Xing Y, et al. Novel insights and current evidence for mechanisms of atherosclerosis: mitochondrial dynamics as a potential therapeutic target. Front Cell Dev Biol. 2021;9:673839. doi:10.3389/ fcell.2021.673839

65. Hughes WE, Beyer AM, Gutterman DD. Vascular autophagy in health and disease. Basic Res Cardiol. 2020;115:41. doi:10.1007/ s00395-020-0802-6

66. Simons M, Gordon E, Claesson-Welsh L. Mechanisms and regulation of endothelial VEGF receptor signalling. Nat Rev Mol Cell Biol. 2016;17:611-625. doi:10.1038/nrm.2016.87

67. Guo D, Wang Q, Li C, Wang Y, Chen X. VEGF stimulated the angiogenesis by promoting the mitochondrial functions. Oncotarget. 2017;8:77020-77027. doi:10.18632/oncotarget.20331

68. Yu S, Zhang L, Liu C, Yang J, Zhang J, Huang L. PACS2 is required for ox-LDL-induced endothelial cell apoptosis by regulating mitochondria-associated ER membrane formation and mitochondrial $\mathrm{Ca}(2$ +) elevation. Exp Cell Res. 2019;379:191-202. doi:10.1016/j. yexcr.2019.04.002

69. Nasi M, De Gaetano A, Bianchini E, et al. Mitochondrial damageassociated molecular patterns stimulate reactive oxygen species production in human microglia. Mol Cell Neurosci. 2020;108:103538. PMID: 32828963. doi:10.1016/j.mcn.2020.103538.

70. Napoli C, Benincasa G, Donatelli F, Ambrosio G. Precision medicine in distinct heart failure phenotypes: focus on clinical epigenetics. $\mathrm{Am}$ Heart J. 2020;224:113-128. doi:10.1016/j.ahj.2020.03.007

71. Costa D, Scognamiglio M, Fiorito C, Benincasa G, Napoli C. Genetic background, epigenetic factors and dietary interventions which influence human longevity. Biogerontology. 2019;20(5):605-626. PMID: 31309340. doi:10.1007/s10522-019-09824-3

72. Schiano C, Benincasa G, Infante T, et al. Integrated analysis of DNA methylation profile of HLA-G gene and imaging in coronary heart disease: pilot study. PLoS One. 2020;15(8):e0236951. PMID: 32790754; PMCID: PMC7425923. doi:10.1371/journal. pone. 0236951

73. Jones AC, Irvin MR, Claas SA, Arnett DK. Lipid phenotypes and DNA methylation: a review of the literature. Curr Atheroscler Rep. 2021;23(11):71. PMID: 34468868. doi:10.1007/s11883-021-00965-w

74. Napoli C, Infante T, Casamassimi A. Maternal-foetal epigenetic interactions in the beginning of cardiovascular damage. Cardiovasc Res. 2011;92:367-374.

75. Napoli C, Crudele V, Soricelli A, et al. Primary prevention of atherosclerosis: a clinical challenge for the reversal of epigenetic mechanisms? Circulation. 2012;125:2363-2373.

76. Collins S. Excess mortality from causes other than influenza and pneumonia during influenza epidemics. Public Health Rep. 1932;47:2159-2179.

77. Bucciarelli V, Nasi M, Bianco F, et al. Depression pandemic and cardiovascular risk in the COVID-19 era and long COVID syndrome: gender makes a difference. Trends Cardiovasc Med. 2021. doi:10.1016/j.tcm.2021.09.009

78. Brooks SK, Webster RK, Smith LE, et al. The psychological impact of quarantine and how to reduce it: rapid review of the evidence. Lancet. 2020;395:912-920. doi:10.1016/S0140-6736(20)30460-8

79. Louvardi M, Pelekasis P, Chrousos G, Darviri C. Mental Health in chronic disease patients during the COVID-19 Quarantine in Greece. Palliat Support Care. 2020;1-17. doi:10.1017/S1478951520000528 
80. Coromilas EJ, Kochav S, Goldenthal I, et al. Worldwide survey of COVID-19-associated arrhythmias. Circ Arrhythm Electrophysiol. 2021;14(3):e009458. doi:10.1161/CIRCEP.120.009458

81. Mattioli AV, Nasi M, Cocchi C, Farinetti A. COVID 19 outbreak: impact of the quarantine-induced stress on cardiovascular disease risk burden. Future Cardiol. 2020. doi:10.2217/fca-2020-0055
82. Silva Andrade B, Siqueira S, de Assis Soares WR, et al. LongCOVID and post-COVID health complications: an up-to-date review on clinical conditions and their possible molecular mechanisms. Viruses. 2021;13(4):700. PMID: 33919537; PMCID: PMC8072585. doi:10.3390/v13040700

\section{Publish your work in this journal}

Vascular Health and Risk Management is an international, peerreviewed journal of therapeutics and risk management, focusing on concise rapid reporting of clinical studies on the processes involved in the maintenance of vascular health; the monitoring, prevention and treatment of vascular disease and its sequelae; and the involvement of metabolic disorders, particularly diabetes. This journal is indexed on PubMed Central and MedLine. The manuscript management system is completely online and includes a very quick and fair peerreview system, which is all easy to use. Visit http://www.dovepress. com/testimonials.php to read real quotes from published authors. 